\title{
VERTEX EXCITED SURFACE WAVES ON ONE FACE OF A RIGHT ANGLED WEDGE*
}

\author{
BY \\ S. N. KARP AND F. C. KARAL, JR. \\ Institute of Mathematical Sciences, New York University
}

\begin{abstract}
The problem of the propagation of electromagnetic waves by a magnetic line dipole source located at the corner of a right angled wedge is considered. It is assumed that an impedance or mixed boundary condition is prescribed on one of the wedge surfaces and that a homogeneous boundary condition is prescribed on the other. The impedance boundary condition is such that surface waves are generated. The amplitude of the surface wave generated is determined. A comparison is made between the magnitude of the surface wave for this problem and that of a magnetic-line dipole source located at the corner of a right angled wedge with the same impedance boundary condition prescribed on both* surfaces. The far field amplitude of the radiated electromagnetic field is also given as an elementary function of the angle of observation.
\end{abstract}

1. Introduction. The problem of the propagation of electromagnetic waves produced by a magnetic line dipole source located at the corner of a right angled wedge is considered. It is assumed that an impedance or mixed boundary condition is prescribed on one of the wedge surfaces and that a homogeneous boundary condition is prescribed on the other. The impedance boundary condition is such that surface waves are generated. The amplitude of the surface wave is determined. A comparison is made between the magnitude of the surface wave for this problem and that of a magnetic-line dipole source located at the corner of a right angled wedge with the same impedance boundary condition prescribed on both surfaces. The latter problem has already been treated by the authors (see Karp and Karal [3]). The comparison of the surface waves for the two different configurations is made on the assumption that the sources have the same strength. The far field representation of the radiated electromagnetic field is also given.

The impedance boundary condition prescribed in the present problem is of the form

$$
\frac{1}{r} \frac{\partial u}{\partial \theta}-\lambda u=0 \quad \theta=\alpha
$$

where $u$ is the $z$ component of the magnetic vector, $r$ and $\theta$ are the usual polar coordinates, $\alpha$ is the wedge angle, and $\lambda$ is a constant characteristic of the surface. The value of $\lambda$ is given by

$$
\lambda=+i \omega \epsilon Z=+i \omega \epsilon(R-i X)
$$

where $\epsilon$ is the permittivity of free space, $\omega$ is the angular frequency and $Z, R$ and $X$ are the impedance, resistance and reactance of the surface, respectively. It is well known that for homogeneous media of large finite conductivity, $R$ and $X$ are positive in sign, small in magnitude and approximately equal. For corrugated or dielectric-coated surfaces,

*Received July 13, 1959. The research reported in this paper has been sponsored by the Electronics Research Directorate of the Air Force Cambridge Research Center, Air Research and Development Command, under Contract No. AF 19(604)1717.

*See Ref. [3] below. 
$R$ and $X$ are positive in sign and $R$ is much smaller than $X$. Hence $X$ is positive in either case and Eq. (1.2) implies that

$$
\operatorname{Re} \lambda>0 .
$$

For illustrations of these boundary conditions see the Introduction of Refs. [3] and [7] where the appropriate references are cited. The condition $R \ll X$ is of course the most important special case insofar as surface waves are concerned.

The problem we treat is not separable because of the mixed boundary conditions. This difficulty can be overcome by the introduction of an auxiliary function which is a linear combination of the magnetic-field and its cartesian derivatives. The auxiliary function is chosen in such a way that it satisfies the wave equation and simple homogeneous boundary conditions. Once the auxiliary function is obtained, the original field can be determined by solving a partial differential equation. This idea is due to Stoker [6] and Lewy [4] who studied problems in water wave theory. Similar ideas have been employed by the authors in the solution of diffraction problems arising in electromagnetic theory. See [1], [2], [3] and [7].

Section 2 contains the exact solution of the problem stated in the preceding paragraphs. The limiting cases of small and large $\lambda / k$ are studied and simplified expressions for the surface waves in both cases are given. A comparison between these results and those obtained in the problem of a line source located at the tip of a right angled wedge with the same impedance boundary condition prescribed on both surfaces is made. In Sec. 3 we discuss the radiated far field and obtain simple expressions for arbitrary values of $\lambda$.

2. Solution. Consider the right angled wedge $y=0, x>0$ and $x=0, y<0$, and suppose there is a magnetic-line dipole located at the origin $x=0, y=0$. (See Fig. 1.) The boundary conditions on the surface are given by

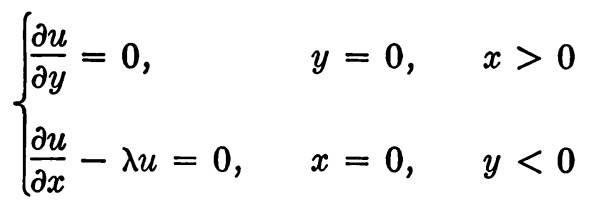

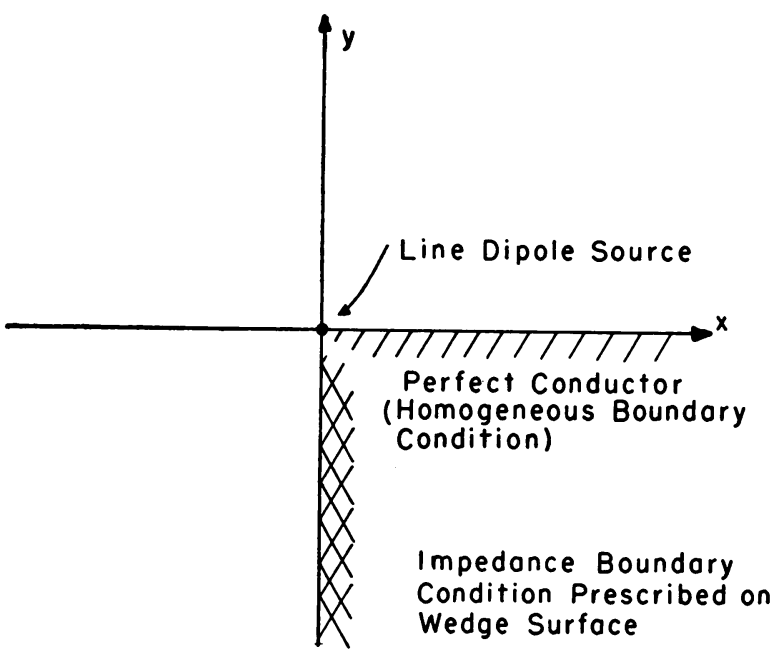


where $u$ is the magnetic vector $H_{z}$ and $\lambda$ is a constant related to the material comprising the surface. We wish to solve the time reduced Maxwell's equations, subject to the prescribed conditions, and obtain the amplitude of the surface wave propagated on the surface $x=0, y<0$.

The time dependent form of Maxwell's equations is

$$
\left\{\begin{array}{l}
\operatorname{curl} \mathbf{H}=-i \omega \epsilon \mathbf{E}, \\
\operatorname{curl} \mathbf{E}=i \omega u \mathbf{H},
\end{array}\right.
$$

where $\mathbf{E}$ and $\mathbf{H}$ are the electric and magnetic field intensities, and $\epsilon$ and $u$ are the permittivity and magnetic permeability of free space. We assume the time dependence to be of the form $\exp (-i \omega t)$. Because of the geometry, the field produced is independent of $z$ and hence the field is completely determined by the value of $H_{z}$. We have

$$
H_{x}=H_{y}=E_{z}=0
$$

and

$$
\left[\begin{array}{l}
E_{x}=-\frac{1}{i \omega \epsilon} \frac{\partial H_{z}}{\partial y} \\
E_{y}=+\frac{1}{i \omega \epsilon} \frac{\partial H_{z}}{\partial x} .
\end{array}\right.
$$

The field component $H_{3} \equiv u$ satisfies the equation

$$
\left(\nabla^{2}+k^{2}\right) u=-4 \pi \delta(x-0) \delta(y-0),
$$

where $\nabla^{2}$ is the rectangular Laplacian, $k$ is the propagation constant of free space and $\delta$ is the Dirac delta function. Therefore, the mathematical problem reduces to that of solving the inhomogeneous wave equation (2.5) subject to the boundary conditions given by (2.1). In addition to the prescribed boundary conditions, we require that the far field be outgoing and that the total magnetic field, excluding the source, be finite everywhere.

Let us make the substitution

$$
v=\frac{\partial u}{\partial x}-\lambda u .
$$

Then $v$ satisfies the following equation:

$$
\left(\nabla^{2}+k^{2}\right) v=0, \quad x^{2}+y^{2} \neq 0, \quad 0 \leq \theta \leq \frac{3 \pi}{2},
$$

subject to the simple conditions

$$
\left\{\begin{array}{l}
\text { (1) } \frac{\partial v}{\partial y}=0 \quad y=0, \quad x>0 \\
\text { (2) } v=0 \quad x=0, \quad y<0 \\
\text { (3) Outgoing waves at infinity. }
\end{array}\right.
$$

If the problem for $v$ can be solved, then we can obtain the solution for the problem involving $u$ since (2.6) can be integrated. 
A particular solution of $(2.6)$ is

$$
u_{p}(x, y)=-\exp (\lambda \xi) \int_{x}^{\infty} \exp (-\lambda x) v(\xi, y) d \xi .
$$

The range of integration is dictated by the condition that $\operatorname{Re} \lambda>0$. It is important to point out that the function $v$ does not have to be finite at the origin. Thus we should first introduce all radiating solutions, no matter how singular at the origin, that satisfy the appropriate boundary conditions for $v$. Out of this class of solutions we finally select only those that yield an everywhere finite value of the total magnetic-field (excluding the source) in the original physical problem involving $u$. By employing the method of separation of variables, we find that the most general representation for $v$ which satisfies conditions (2.8) is of the form $\sum a_{n} H_{(2 n+1) / 3}^{(1)}(k r) \cos [(2 n+1) / 3] \theta$, where $n$ is an integer and $H_{(2 n+1) / 3}^{(1)}(k r)$ is a Hankel function of the first kind of order $(2 n+1) / 3$. Since $v$ cannot be too singular, the only admissible functions of this class are

$$
v(x, y)=c_{0} H_{1}^{(1)}(k r) \cos \theta+c_{1} H_{1 / 3}^{(1)}(k r) \cos \frac{\theta}{3} \text {. }
$$

The above expression for $v$ is a wave function, defined, by means of (2.7), in the angular sector $0 \leq \theta \leq 3 \pi / 2$. We note, however, that we can extend the range of definition of $v$ to include the angular sector $0 \leq \theta \leq 2 \pi$ by means of the reflection principle since $v$ vanishes on the negative $y$-axis. This extension is necessary since the range of integration of the particular solution (2.9) extends into the fourth quadrant for negative values of $y$. Using this extended definition of $v$, the particular solution (2.9) becomes

$$
u_{p}(x, y)=-\exp (\lambda \xi) \int_{x}^{\infty} \exp (-\lambda \xi)\left\{c_{0} H_{1}^{(1)}(k r) \cos \theta+c_{1} H_{1 / 3}^{(1)}(k r) \cos \frac{\theta}{3}\right\} d \xi .
$$

The above result, though well defined, does not satisfy all the conditions of the problem since it is not regular across the line $y=0, x<0$. This difficulty can be overcome by adding a complementary solution, $u_{c}$ say, of the inhomogeneous equation (2.6) to (2.11). We then obtain the representation

$$
\begin{array}{r}
u(x, y)=-\exp (\lambda x) \int_{x}^{\infty} \exp (-\lambda \xi)\left\{c_{0} H_{1}^{(1)}(k r) \cos \theta+c_{1} H_{1 / 3}^{(1)}(k r) \cos \frac{\theta}{3}\right\} d \xi \\
+\left[\begin{array}{cc}
c_{2} \exp \left[\lambda x-i\left(k^{2}+\lambda^{2}\right)^{1 / 2} y\right], & y<0 \\
0 \quad, & y>0
\end{array}\right\},
\end{array}
$$

where $r=\left(\xi^{2}+y^{2}\right)^{1 / 2}, \theta=\arctan \xi / y$ and $0 \leq \theta \leq 2 \pi$. The complementary solution $u_{c}$, which is indicated in square brackets in (2.12), is obviously not a wave function since it is discontinuous across the line $y=0$. However, although each solution separately is discontinuous together with its $y$-derivative along the negative $x$-axis, the solution $u=u_{p}+u_{c}$ of (2.12) must be regular there. From this fact we can derive two relations between the constants $c_{0}, c_{1}$ and $c_{2}$ by calculating the discontinuities of $u$ and $\partial u / \partial y$ across the negative $x$-axis and setting the discontinuities equal to zero. These calculations completely solve the problem because the constant $c_{0}$ is related to the source strength and is known. Hence there are a sufficient number of equations to determine all of the unknown constants. We note that the form of the term in brackets in (2.12) is that of a surface wave with an amplitude given by $c_{2}$.

Before proceeding to the calculation of the discontinuities and the value of the 
constants $c_{1}$ and $c_{2}$, we first simplify the expression for $u$ and then determine $\partial u / \partial y$. By using the relation

$$
\frac{\partial}{\partial x} H_{0}^{(1)}(k r)=-k H_{1}^{(1)}(k r) \cos \theta
$$

and integrating by parts, (2.12) becomes

$$
\begin{aligned}
& u(x, y)=-\frac{c_{0}}{k}\left\{H_{0}^{(1)}(k r)-\lambda \exp (\lambda x) \int_{x}^{\infty} \exp (-\lambda \xi) H_{0}^{(1)}(k r) d \xi\right\} \\
&-c_{1} \exp (\lambda x) \int_{x}^{\infty} \exp (-\lambda \xi) H_{1 / 3}^{(1)}(k r) \cos \frac{\theta}{3} d \xi \\
&+\left[\begin{array}{cr}
c_{2} \exp \left[\lambda x-i\left(k^{2}+\lambda^{2}\right)^{1 / 2} y\right], & y<0 \\
0 \quad, & y>0
\end{array}\right] .
\end{aligned}
$$

At this stage we can determine $c_{0}$ since we recognize the Hankel function $H_{0}^{(1)}(k r)$ as being the Green's function for a line source. The solution of (2.5) for a simple point source located at the origin is given by the well-known result

$$
G(r, \theta ; 0,0)=i \pi H_{0}^{(1)}(k r) \text {. }
$$

Hence the proper normalization for a point source is obtained provided we let

$$
c_{0}=-i \pi k \text {. }
$$

We next determine $\partial u / \partial y$. We have

$$
\begin{aligned}
& u_{y}(x, y) \equiv \frac{\partial u}{\partial y}(x, y)=-\frac{c_{0}}{k}\left[\frac{\partial}{\partial y} H_{0}^{(1)}(k r)-\lambda \exp (\lambda x) \int_{x}^{\infty} \exp (-\lambda \xi) \frac{\partial}{\partial y}\left\{H_{0}^{(1)}(k r)\right\} d \xi\right] \\
&- c_{1} \exp (\lambda x) \int_{x}^{\infty} \exp (-\lambda \xi) \frac{\partial}{\partial y}\left\{H_{1 / 3}^{(1)}(k r) \cos \frac{\theta}{3}\right\} d \xi \\
&+\left[\begin{array}{cr}
-c_{2} i\left(k^{2}+\lambda^{2}\right)^{1 / 2} \exp \left[\lambda x-i\left(k^{2}+\lambda^{2}\right)^{1 / 2} y\right], & y<0 \\
0 \quad, & y>0
\end{array}\right],
\end{aligned}
$$

where we have taken the derivative inside the integral sign. By using the following relations

$$
\begin{aligned}
\frac{\partial}{\partial y} H_{0}^{(1)}(k r) & =-k H_{1}^{(1)}(k r) \sin \theta, \\
\frac{\partial}{\partial y}\left\{H_{1 / 3}^{(1)}(k r) \cos \frac{\theta}{3}\right\}-\frac{\partial}{\partial x}\left\{H_{1 / 3}^{(1)}(k r) \sin \frac{\theta}{3}\right\} & =k \exp [i(2 \pi / 3)] H_{2 / 3}^{(1)}(k r) \sin \frac{2 \theta}{3}
\end{aligned}
$$

and integrating by parts we obtain

$$
\begin{aligned}
u_{y}(x, y)=-\frac{c_{0}}{k} & \left\{-k H_{1}^{(1)}(k r) \sin \theta+k \lambda \exp (\lambda x) \int_{x}^{\infty} \exp (-\lambda \xi) H_{1}^{(1)}(k r) \sin \theta d \xi\right\} \\
& +c_{1} H_{1 / 3}^{(1)}(k r) \sin \frac{\theta}{3}-c_{1} \lambda \exp (\lambda x) \int_{x}^{\infty} \exp (-\lambda \xi) H_{1 / 3}^{(1)}(k r) \sin \frac{\theta}{3} d \xi \\
& -c_{1} k \exp [i(2 \pi / 3)] \exp (\lambda x) \int_{x}^{\infty} \exp (-\lambda \xi) H_{2 / 3}^{(1)}(k r) \sin \frac{2 \theta}{3} d \xi \\
& +\left[\begin{array}{cr}
-c_{2} i\left(k^{2}+\lambda^{2}\right)^{1 / 2} \exp \left[\lambda x-i\left(k^{2}+\lambda^{2}\right)^{1 / 2} y\right], & y<0 \\
0 & ,
\end{array}\right] .
\end{aligned}
$$


It was noted earlier that the constants $c_{1}$ and $c_{2}$ can be determined by requiring that $u$ and its derivatives be continuous throughout the region of physical space $(0 \leq \theta \leq 3 \pi / 2)$. This is equivalent to calculating the discontinuities of $u$ and $\partial u / \partial y$ across the negative $x$-axis and setting the discontinuities equal to zero. Hence for $u(x, y)$ to be a wave function, we require that the following jump conditions be satisfied:

$$
\left\{\begin{aligned}
(\mathrm{I})[u] & \equiv\left\{u\left(x, 0^{+}\right)-u\left(x, 0^{-}\right)\right\}=0, & & x<0 \\
(\mathrm{II})\left[u_{y}\right] & \equiv\left\{u_{y}\left(x, 0^{+}\right)-u_{y}\left(x, 0^{-}\right)\right\}=0, & & x<0 .
\end{aligned}\right.
$$

The first jump condition yields immediately

$$
c_{2}=-\frac{3}{2} c_{1} I_{1 / 3}(k, \lambda)
$$

where we have used the notation

$$
I_{\nu}(k, \lambda)=\int_{0}^{\infty} \exp (-\lambda \xi) H_{\nu}^{(1)}(k|\xi|) d \xi .
$$

We note that

$$
\begin{aligned}
I_{\nu}(k, \lambda) & =\frac{i}{\sin \nu \pi} \frac{\exp [-i \nu(\pi / 2)]}{k^{\nu}\left(\lambda^{2}+k^{2}\right)^{1 / 2}} \\
\cdot & \left\{\exp [-i \nu(\pi / 2)]\left(\left[\lambda^{2}+k^{2}\right]^{1 / 2}-\lambda\right)^{\nu}-\exp [i \nu(\pi / 2)]\left(\left[\lambda^{2}+k^{2}\right]^{1 / 2}+\lambda\right)^{\nu}\right\},
\end{aligned}
$$

where $-1>\operatorname{Re} \nu<+1$. (See Magnus and Oberhettinger [5].) The second jump condition requires more careful treatment. If we substitute (2.20) into jump condition (II) and use (2.16) we obtain

$$
\begin{aligned}
{\left[u_{y}\right]=\operatorname{Lim}_{y \rightarrow+0} 2 \pi i k \lambda } & \exp (\lambda x) \int_{x}^{\infty} \exp (-\lambda \xi) H_{1}^{(1)}\left(k\left[\xi^{2}+y^{2}\right]^{1 / 2}\right) \frac{y}{\left(\xi^{2}+y^{2}\right)^{1 / 2}} d \xi \\
& +c_{1} \lambda \exp (\lambda x) \sin \frac{2 \pi}{3} \int_{0}^{\infty} \exp (-\lambda \xi) H_{1 / 3}^{(1)}(k|\xi|) d \xi \\
& +c_{1} k \exp [i(2 \pi / 3)] \exp (\lambda x) \sin \frac{4 \pi}{3} \int_{0}^{\infty} \exp (-\lambda \xi) H_{2 / 3}^{(1)}(k|\xi|) d \xi \\
& +c_{2} i\left(k^{2}+\lambda^{2}\right)^{1 / 2} \exp (\lambda x)=0 .
\end{aligned}
$$

Let us consider the expression

$$
J=\lim _{y \rightarrow+0} \int_{x}^{\infty} \exp (-\lambda \xi) H_{1}^{(1)}\left(k\left[\xi^{2}+y^{2}\right]^{1 / 2}\right) \frac{y}{\left(\xi^{2}+y^{2}\right)^{1 / 2}} d \xi .
$$

The integrand in (2.26) tends to zero with $y$ except in the neighborhood of $\xi=0$. Hence the only possible contribution occurs when $\xi$ lies in an interval $-\epsilon$ to $\epsilon$, where $\epsilon$ is arbitrarily small. Since we assume that $y$ is already small, we can use the asymptotic representations for the exponential and Hankel functions appearing in the integrand. Hence

$$
J=\frac{-2 i}{\pi k} \operatorname{Lim}_{y \rightarrow+0} \int_{-\epsilon}^{\epsilon} \frac{y}{\xi^{2}+y^{2}} d \xi .
$$

If we make the substitution $\xi=y t$, the above integral becomes

$$
J=-\frac{2 i}{\pi k} \int_{-\infty}^{\infty} \frac{d t}{1+t^{2}}=-2 \frac{i}{k} .
$$


Substituting this result into (2.25) and using the notation defined in (2.23), the second jump condition becomes

$$
\begin{aligned}
+4 \pi \lambda+c_{1} \lambda \sin \frac{2 \pi}{3} & I_{1 / 3}(k, \lambda) \\
& +c_{1} k \exp [i(2 \pi / 3)] \sin \frac{4 \pi}{3} I_{2 / 3}(k, \lambda)+c_{2} i\left(k^{2}+\lambda^{2}\right)^{1 / 2}=0 .
\end{aligned}
$$

By substituting (2.22) into (2.29) we find $c_{1}$. We obtain

$$
c_{1}=-\frac{4 \pi}{3^{1 / 2}} \frac{2 \lambda}{\left(\lambda-i 3^{1 / 3}\left[k^{2}+\lambda^{2}\right]^{1 / 2}\right) I_{1 / 3}-k \exp [i(2 \pi / 3)] I_{2 / 3}}
$$

and

$$
c_{2}=+23^{1 / 2} \pi \frac{2 \lambda I_{1 / 3}}{\left(\lambda-3^{1 / 2} i\left[k^{2}+\lambda^{2}\right]^{1 / 2}\right) I_{1 / 3}-k \exp [i(2 \pi / 3)] I_{2 / 3}} .
$$

The value of $c_{2}$ is the amplitude of the surface wave propagated along the wedge surface.

We next determine the values of $c_{1}$ and $c_{2}$ for small and large values of $\lambda / k$. When $\lambda / k$ is small it is easily shown that

$$
\begin{aligned}
& I_{1 / 3} \approx \frac{1}{k}\left(1-i 3^{-1 / 2}\right)=\frac{2}{k} 3^{-1 / 2} \exp [-i(\pi / 6)] \\
& I_{2 / 3} \approx \frac{1}{k}\left(1-i 3^{1 / 2}\right)=\frac{2}{k} \exp [-i(\pi / 3)]
\end{aligned}
$$

and the approximate expressions for $c_{1}$ and $c_{2}$ become

$$
\begin{aligned}
& c_{1} \approx \frac{2 \pi \lambda}{3^{1 / 2}} \exp [-i(\pi / 3)] \\
& c_{2} \approx 2 \pi i\left(\frac{\lambda}{k}\right) .
\end{aligned}
$$

When $\lambda / k$ is large we find that

$$
\begin{aligned}
& I_{1 / 3} \approx-i \frac{2}{3^{1 / 2}}\left(\frac{2}{k}\right)^{1 / 3} \frac{1}{\lambda^{2 / 3}} \\
& I_{2 / 3} \approx-i \frac{2}{3^{1 / 2}}\left(\frac{2}{k}\right)^{2 / 3} \frac{1}{\lambda^{1 / 3}}
\end{aligned}
$$

and the approximate expressions for $c_{1}$ and $c_{2}$ become

$$
\begin{aligned}
& c_{1} \approx \pi k \exp [-i(\pi / 6)]\left(\frac{2 \lambda}{k}\right)^{2 / 3} \\
& c_{2} \approx 23^{1 / 2} \pi \exp [i(\pi / 3)] .
\end{aligned}
$$

It is of interest to compare the amplitude of the surface wave generated in this problem with that generated by a line source located at the tip of a right-angled wedge with the same impedance boundary condition prescribed on both surfaces. The latter problem has previously been solved [3] and the amplitude of the surface wave propagated on the wedge surface $x=0, y<0$ is given by 
$c_{3}=43^{1 / 2} \pi \frac{\lambda I_{2 / 3}-k \exp [i(\pi / 3)] I_{1 / 3}}{\left\{\left[i\left(k^{2}+\lambda^{2}\right)^{1 / 2}-\lambda 3^{1 / 2}\right] I_{2 / 3}+3^{1 / 2} k \exp [i(\pi / 3)] I_{1 / 3}\right\}}$

$$
\frac{\lambda}{\left[i\left(k^{2}+\lambda^{2}\right)^{1 / 2}-\lambda\right]}
$$

When $\lambda / k$ is small we find that

$$
c_{3} \approx 2 \pi i\left(\frac{\lambda}{k}\right)
$$

and when $\lambda / k$ is large

$$
c_{3} \approx \frac{4 \pi}{(1-i)\left(1-i 3^{1 / 2}\right)}=2 \pi\left(\frac{3}{2}\right)^{1 / 2} \exp [i(5 \pi / 12)] .
$$

Hence, when the value of $\lambda / k$ is small, the ratio of the amplitude of the surface wave on a wedge with an impedance boundary condition on one surface to that on both surfaces is given by unity. When the value of $\lambda / k$ is large, the ratio is given by $2^{1 / 2}$. In the limit $\lambda / k \rightarrow \infty$ therefore, the total energy that goes into surface waves in our present configuration is the same as the energy so utilized when both faces of the wedge can support surface waves.

3. Determination of the radiated far field. We have already obtained the exact solution for a line source located at the tip of a right-angled wedge with an impedance type boundary condition prescribed on one surface. The exact solution as given by (2.14), however, is complicated and it is desirable to find a simple expression for the radiated far field. From (2.6) and (2.10) we have

$$
v=\frac{\partial u}{\partial x}-\lambda u
$$

and

$$
v=c_{0} H_{1}^{(1)}(k r) \cos \theta+c_{1} H_{1 / 3}^{(1)}(k r) \cos \frac{\theta}{3} .
$$

When $k r$ is large (3.2) becomes

$v=c_{0}\left(\frac{2}{\pi k r}\right)^{1 / 2} \exp \{i[k r-(3 \pi / 4)]\} \cos \theta$

$$
+c_{1}\left(\frac{2}{\pi k r}\right)^{1 / 2} \exp \{i[k r-(5 \pi / 12)]\} \cos \frac{\theta}{3} .
$$

We expect the far field of the $z$-component of the magnetic field vector to have a similar form, except that it contains surface wave terms which are appreciable near the wedge surface $x=0, y<0$. Hence for a fixed $\theta$, as $r \rightarrow \infty$ we have

$$
u=\left[\frac{l(\theta)}{r^{1 / 2}} \exp (i k r)+0\left(\frac{1}{r^{3 / 2}}\right)\right], \quad \theta \neq 3 \pi / 2,
$$

where $l(\theta)$ is unknown. Now

$$
\frac{\partial}{\partial x}=\cos \theta \frac{\partial}{\partial r}-\frac{1}{r} \sin \theta \frac{\partial}{\partial \theta} .
$$


If we substitute (3.3), (3.4) and (3.5) into (3.1), and keep terms of order $r^{-1 / 2}$, we obtain

$$
l(\theta)=\left(\frac{2}{\pi k}\right)^{1 / 2} \frac{c_{0} \exp [-i(3 \pi / 4)] \cos \theta+c_{1} \exp [-i(5 \pi / 12)] \cos \frac{\theta}{3}}{i k \cos \theta-\lambda} .
$$

Hence the desired radiated field is given by

$u(r, \theta)=\left(\frac{2}{\pi k r}\right)^{1 / 2} e^{i k r} \frac{c_{0} \exp [-i(3 \pi / 4)] \cos \theta+c_{1} \exp [-i(5 \pi / 12)] \cos \frac{\theta}{3}}{i k \cos \theta-\lambda}+0\left(\frac{1}{r^{3 / 2}}\right)$

where $c_{0}, c_{1}$ and $c_{2}$ are given by (2.16), (2.30) and (2.31), respectively.

\section{REFERENCES}

1. F. C. Karal and S. N. Karp, Diffraction of a skew plane electromagnetic wave by an absorbing right angled wedge, Communs. Pure Appl. Math. 11, No. 4, (Nov. 1958); also, N. Y. U., Inst. Math. Sci. Div. EM Research, Research Rept. No. EM-111, Feb. 1958

2. S. N. Karp, Two dimensional Green's function for a right angled wedge under an impedance boundary condition, N. Y. U., Inst. Math. Sci., Div. EM Res. Rept. No. EM-129

3. S. N. Karp and F. C. Karal, Surface waves on a right angled wedge, N. Y. U., Inst. Math. Sci., Div. EM Res., Research Rept. EM-116, Aug. 1958. Condensed version: 1958 IRE Wescon Convention Record, Part I, 101-103, Communs. Pure Appl. Math. 12, 3 (1959) (amended title: Vertex excited surface waves on both faces of a right angled wedge)

4. H. Lewy, Waves on sloping beaches, Bull. AMS 52, 737 (1946)

5. W. Magnus and F. Oberhettinger, Formulas and theorems for the special functions of mathematical physics, 2nd ed., Berlin, Springer, 1948

6. J. J. Stoker, Surface waves in water of variable depth, Quart. Appl. Math. 5, 1 (1947)

7. F. C. Karal and S. N. Karp, Diffraction of a plane wave by a right angled wedge which sustains surface waves on one face, N. Y. U., Inst. Math. Sci., Div. EM Res., Research Rept. No. EM-123, Jan. 1959 